\title{
Imunoterapia em Oncologia em uma Cidade do Interior de Minas Gerais: Análise da Década 2010-2019
}

doi: https://doi.org/10.32635/2176-9745.RBC.2020v66n4.1074

\author{
Immunotherapy in Oncology in a Countryside City of Minas Gerais: Analysis of the decade 2010-2019 \\ Inmunoterapia en Oncología en una Ciudad del Interior de Minas Gerais: Analisis de la Década 2010-2019
}

\author{
Camila Silveira Campos ${ }^{1}$; Fernanda Lopes Bessa ${ }^{2}$; Isabelle Fernanda Ladeira de Melo ${ }^{3}$; Luana França Esteves ${ }^{4}$; Marcella Rodrigues \\ Messias ; Sávia Gabriela Tameirão Pedroso Gregório de Souza'; Priscilla Brunelli Pujatti ${ }^{7}$
}

\section{RESUM0}

Introduçáo: Nos últimos anos, a terapia sistêmica em oncologia sofreu mudanças substanciais e a imunoterapia destacou-se como forma de tratamento adjuvante. No Brasil, as diferenças do acesso a essa terapêutica podem ser observadas de maneira relevante e ainda não há amplo conhecimento sobre o seu uso nas diferentes Regiôes do país. Objetivo: Analisar o panorama da utilização de imunoterapia no tratamento oncológico em Barbacena-MG na última década. Método: Estudo descritivo de centro único. Os pacientes maiores de 18 anos, diagnosticados com câncer e que utilizaram imunoterápicos no período de 2010 a 2019, foram selecionados por meio de consulta ao Registro Hospitalar de Câncer, e as informaçóes constantes nesse sistema, bem como prontuários, foram utilizadas para obtençáo de dados sociodemográficos e clínicos. Resultados: A imunoterapia foi utilizada no tratamento de 90 pacientes (4,9\%), totalizando 95 tratamentos. Entre os medicamentos utilizados, destacaram-se o Bacillus Calmette-Guérin (BCG), o trastuzumabe e o imatinibe. Observou-se um aumento na utilizaçáo de imunoterápicos ao longo da década, bem como uma maior variedade de tratamentos realizados. O imatinibe e o pazopanibe obtiveram maior porcentagem de óbitos durante o tratamento e $77,9 \%$ dos medicamentos utilizados foram custeados pelo Sistema Único de Saúde (SUS). O tempo de tratamento da maioria dos pacientes se manteve próximo a dez meses, sendo menor para aqueles que se trataram com BCG. Conclusáo: $\mathrm{O}$ uso da imunoterapia em oncologia em Barbacena apresentou um avanço no período de 2010 a 2019, tanto no quantitativo quanto na variedade de tratamentos, com incorporaçáo de novos medicamentos.

Palavras-chave: Neoplasias/terapia; Imunoterapia; Brasil.

\section{ABSTRACT}

Introduction: In recent years, systemic therapy in oncology has undergone substantial changes and immunotherapy has spread out as a form of adjuvant treatment. In Brazil, differences of access to this therapy are relevant and there is still no extensive knowledge about the use of immunotherapy in different regions of the country. Objective: To analyze the panorama of immunotherapy in cancer treatment in Barbacena-MG in the last decade. Method: Single-centre descriptive study. Patients over 18 years diagnosed with cancer who used immunotherapies in the period from 2010 to 2019 were selected by reviewing the Hospital Cancer Registry and the information contained in this system, as well as charts, were used to obtain sociodemographic and clinical data. Results: Immunotherapy was used to treat 90 patients (4.9\%), totalling 95 treatments. Bacillus Calmette-Guérin (BCG), trastuzumab and imatinib were the drugs most used. There was an increase in the use of immunotherapeutic agents over the decade, as well as in the variety of treatments performed. Imatinib and pazopanib had a higher percentage of deaths during treatment and $77.9 \%$ of the drugs used were funded by the Unified Health System (SUS). Treatment duration for most patients remained close to 10 months, being shorter for those who were treated with BCG. Conclusion: The use of immunotherapy in oncology in Barbacena has grown from 2010 to 2019, in number and variety of treatments, with incorporation of new drugs.

Key words: Neoplasms/therapy; Immunotherapy; Brazil.

\section{RESUMEN}

Introducción: En las últimas décadas han permitido el desarrollo de nuevas modalidades de tratamientos anticancerosos y la inmunoterapia se ha destacado. En Brasil, el acceso desigual a inmunoterapia es relevante y todavía no hay amplio conocimiento sobre su uso en las distintas regiones del país. Objetivo: Analizar el panorama de la inmunoterapia en el tratamiento anticanceroso en Barbacena-MG en la última década. Método: Estudio descriptivo de centro único, con datos del Registro Hospitalario de Cáncer y registros médicos de los pacientes que usaron inmunoterapéuticos en el tratamiento anticanceroso de 2010 a 2019. Resultados: Se utilizó inmunoterapéuticos para tratar a 90 pacientes $(4,9 \%)$, totalizando 95 tratamientos. Entre los medicamentos utilizados, se destacaron Bacillus Calmette-Guérin (BCG), trastuzumab e imatinib. Hubo un aumento en el uso de inmunoterapéuticos en la década, así como una mayor variedad de tratamientos realizados. Imatinib y pazopanib tuvieron un mayor porcentaje de muertes durante el tratamiento y $77,9 \%$ de los medicamentos utilizados fueron financiados por el Sistema Único de Salud (SUS). El tiempo de tratamiento para la mayoría de los pacientes fue diez meses, siendo más corto para el tratamiento con BCG. Conclusión: El uso de la inmunoterapia en oncología en Barbacena ha avanzado en el período de 2010 a 2019, tanto en cantidad como variedad de tratamientos, con incorporación de nuevos medicamentos.

Palabras clave: Neoplasias/terapia; Inmunoterapia; Brasil.

'Fundação José Bonifácio Lafayette de Andrada (FUNJOBE). Faculdade de Medicina de Barbacena (FAME). Barbacena (MG), Brasil. Orcid iD: https://orcid.org/00000002-1090-1801

${ }^{2}$ FUNJOBE. FAME. Barbacena (MG), Brasil. Orcid iD: https://orcid.org/0000-0001-8244-6898

${ }^{3}$ FUNJOBE. FAME. Barbacena (MG), Brasil. Orcid iD: https://orcid.org/0000-0001-6877-1301

${ }^{4}$ FUNJOBE. FAME. Barbacena (MG), Brasil. Orcid iD: https://orcid.org/0000-0002-3135-6734

${ }^{5}$ FUNJOBE. FAME. Barbacena (MG), Brasil. Orcid iD: https://orcid.org/0000-0002-5048-5793

${ }^{6}$ FUNJOBE. FAME. Barbacena (MG), Brasil. Orcid iD: https://orcid.org/0000-0002-9499-8400

${ }^{7}$ FUNJOBE. FAME. Barbacena (MG), Brasil. Instituto Nacional de Câncer José de Alencar Gomes da Silva (INCA). Rio de Janeiro (RJ), Brasil. Orcid iD: https://orcid. org/0000-0002-1165-8049

Endereço para correspondência: Priscilla Brunelli Pujatti. Praça Presidente Antônio Carlos, 8 - São Sebastião. Barbacena (MG), Brasil. CEP $36202-336$. E-mail: pujatti.pb@gmail.com 


\section{INTRODUÇÃO}

O câncer é uma doença crônica de grande prevalência e alta morbimortalidade, sendo atualmente responsável por uma em cada seis mortes no mundo. Mais de 14 milhóes de pessoas desenvolvem essa doença todos os anos e a previsão é que esse número deva subir para mais de 21 milhôes em $2030^{1}$. Estima-se, para o Brasil, a ocorrência de 625 mil novos casos de neoplasias, para cada ano do triênio 2020-2022, segundo o Instituto Nacional de Câncer José de Alencar Gomes da Silva (INCA)².

O tratamento convencional contra o câncer foi, por décadas, a combinação de cirurgia, quimioterapia e/ou radioterapia ${ }^{3}$. Entretanto, nos últimos anos, a terapia sistêmica em oncologia sofreu mudanças substanciais. Os avanços no entendimento de anormalidades genéticas levaram ao desenvolvimento de diferentes terapias-alvo. Alguns novos tratamentos quimioterápicos foram aprovados, e outros, já bem conhecidos, têm sido mais amplamente utilizados. Além disso, uma melhor compreensão da interação entre as células tumorais e o sistema imunológico sustentou o desenvolvimento de imunoterapias ${ }^{4}$.

A imunoterapia promove a estimulação do sistema imunológico por meio do uso de substâncias modificadoras da resposta biológica. A ideia foi originalmente proposta por William Coley em 1893. Em seguida, Paul Ehrlich, em 1909, sugeriu que o sistema imunológico deva ter um papel no embate contra o câncer ${ }^{5,6}$.

A imunoterapia em oncologia pode ser classificada em ativa e passiva, de acordo com as substâncias utilizadas e os seus mecanismos de ação. $\mathrm{Na}$ imunoterapia ativa, constituintes estimulantes e restauradores da função imunológica (imunoterapia inespecífica, a exemplo de modificadores biológicos como o Bacillus Calmette-Guérin - BCG e citocinas) e vacinas de células tumorais (imunoterapia específica) são aplicados com o objetivo de intensificar a resistência ao crescimento tumoral. $\mathrm{Na}$ imunoterapia passiva ou adotiva, anticorpos antitumorais ou células mononucleares exógenas são administrados com a finalidade de proporcionar capacidade imunológica de combate à doença, a exemplo de anticorpos monoclonais ${ }^{3}$.

A utilizaçáo da imunoterapia provocou profundas transformaçóes no tratamento do câncer em todo o mundo ${ }^{7}$. Entretanto, a entrada dos imunoterápicos não se fez da mesma maneira em todos os países, sendo influenciada por diversos fatores, não apenas clínicos e técnico-científicos, mas também mercadológicos ${ }^{8}$. Mais especificamente no Brasil, país de proporçôes continentais e onde a desigualdade socioeconômica também se traduz no acesso a tecnologias em saúde, essas diferenças podem ser observadas entre Estados e entre cidades do mesmo
Estado. Ademais, não há amplo conhecimento sobre o uso dessa terapia nas diferentes Regiôes do país, sendo preciso avaliar sua utilização e amplificar a disseminação de informaçóes acerca do uso. Neste contexto, o objetivo do presente estudo foi analisar o panorama da utilização de imunoterapia no tratamento oncológico em Barbacena-MG na última década.

\section{MÉTODO}

Trata-se de um estudo descritivo que teve como objeto as imunoterapias em oncologia realizadas de 2010 a 2019 na unidade hospitalar que presta atendimento em oncologia em Barbacena-MG. O município é referência regional para atendimento de câncer, abrangendo mais de 14 municípios, o que caracteriza assistência a 225.183 habitantes?.

Os critérios de inclusão foram pacientes oncológicos maiores de 18 anos, diagnosticados com câncer em quaisquer estádios da doença, em uso de imunoterapia para tratamento de neoplasias malignas e tratados entre 2010 e 2019. Foram excluídos pacientes que utilizaram imunoterápicos apenas como moduladores da resposta imune e não como parte do tratamento antineoplásico e aqueles pacientes que estavam em tratamento, mas tiveram diagnóstico em outra unidade.

Os pacientes com câncer e que utilizaram imunoterápicos como parte da terapia foram selecionados por meio de consulta ao Registro Hospitalar de Câncer (RHC), em seu formato eletrônico. Nesse sistema, faz-se uma seleção dos pacientes atendidos, de acordo com o critério desejado, que, no caso, foi a utilização de algum imunoterápico como forma de tratamento. Uma vez selecionados os pacientes, os dados constantes no RHC, bem como o prontuário, foram utilizados para obtenção dos dados sociodemográficos e clínicos, e os seguintes dados de acompanhamento/prognóstico: (I) imunoterápico utilizado; (II) início e término do tratamento; (III) óbito durante o tratamento (se aplicável).

O estudo foi aprovado pelo Comitê de Ética em Pesquisa da Faculdade de Medicina de Barbacena-MG, sob o número CAAE 06670919.8.0000.8307, parecer 3.144.278, de 13 de fevereiro de 2019, e desenvolvido conforme estabelecido na Resoluçáo CNS n. ${ }^{0} 466 / 12^{10}$.

Os dados obtidos por meio dos formulários e prontuários foram transcritos para uma planilha eletrônica e processados no software estatístico Stata versão 9.2. A partir disso, tabelas com frequência absoluta e relativa das variáveis estudadas foram produzidas. Para as variáveis quantitativas, foram calculadas medidas de tendência central, posição e dispersão. 


\section{RESULTADOS}

Na última década, 90 pacientes realizaram tratamento oncológico com imunoterapia no Hospital Ibiapaba. Destes, cinco apresentaram recidivas e foram retratados, totalizando 95 tratamentos. Nesse mesmo período, 1.837 pacientes foram tratados na instituição, sendo, portanto, a imunoterapia utilizada em $4,9 \%$ dos pacientes tratados. As características sociodemográficas dos pacientes estáo apresentadas na Tabela 1. Observou-se maior prevalência de tratamento imunoterápico em pacientes maiores de 61 anos, brancos. Entre os medicamentos utilizados, destacam-se o BCG como modificador biológico, o trastuzumabe como anticorpo monoclonal e o imatinibe como inibidor da proteína quinase.

Tabela 1. Características sociodemográficas de pacientes $(n=90)$ diagnosticados com câncer e que receberam o primeiro tratamento com imunoterápicos na unidade hospitalar para tratamento oncológico, Barbacena-MG, 2010-2019

\begin{tabular}{|c|c|c|c|}
\hline Características & Variáveis & $\mathbf{n}$ & $\%$ \\
\hline Idade (anos) & $\begin{array}{l}29 \text { a } 40 \\
41 \text { a } 50 \\
51 \text { a } 60 \\
61 \text { a } 70 \\
71 \text { a } 80 \\
>81\end{array}$ & $\begin{array}{l}6 \\
11 \\
18 \\
29 \\
18 \\
8\end{array}$ & $\begin{array}{c}6,66 \\
12,21 \\
19,98 \\
32,21 \\
19,98 \\
8,88\end{array}$ \\
\hline Sexo & $\begin{array}{l}\text { Feminino } \\
\text { Masculino }\end{array}$ & $\begin{array}{l}41 \\
49\end{array}$ & $\begin{array}{l}45,56 \\
54,44\end{array}$ \\
\hline Raça & $\begin{array}{l}\text { Branco (a) } \\
\text { Preto (a) } \\
\text { Pardo (a) }\end{array}$ & $\begin{array}{c}60 \\
6 \\
24\end{array}$ & $\begin{array}{c}66,67 \\
6,67 \\
26,67\end{array}$ \\
\hline Escolaridade & $\begin{array}{c}<9 \text { anos de estudo } \\
>9 \text { anos de estudo } \\
\text { Sem informação }\end{array}$ & $\begin{array}{l}33 \\
25 \\
32\end{array}$ & $\begin{array}{l}36,67 \\
27,78 \\
35,56\end{array}$ \\
\hline $\begin{array}{l}\text { Histórico de } \\
\text { alcoolismo }\end{array}$ & $\begin{array}{c}\text { Não } \\
\text { Sim } \\
\text { Sem informação }\end{array}$ & $\begin{array}{l}44 \\
14 \\
32\end{array}$ & $\begin{array}{l}48,89 \\
15,55 \\
35,56\end{array}$ \\
\hline $\begin{array}{l}\text { Histórico de } \\
\text { tabagismo }\end{array}$ & $\begin{array}{c}\text { Não } \\
\text { Sim } \\
\text { Sem informação }\end{array}$ & $\begin{array}{l}34 \\
31 \\
25\end{array}$ & $\begin{array}{l}37,78 \\
34,44 \\
27,78\end{array}$ \\
\hline \multirow{4}{*}{$\begin{array}{c}\text { Imunoterápicos } \\
\text { utilizados }\end{array}$} & $\begin{array}{c}\text { MODIFICADORES } \\
\text { BIOLÓGICOS } \\
\text { BCG } \\
\text { Interferon alfa-2B }\end{array}$ & $\begin{array}{l}58 \\
51 \\
7\end{array}$ & $\begin{array}{c}61,05 \\
53,68 \\
7,37\end{array}$ \\
\hline & $\begin{array}{l}\text { ANTICORPOS } \\
\text { MONOCLONAIS } \\
\text { Trastuzumabe } \\
\text { Brentuximabe } \\
\text { Rituximabe } \\
\text { Bevacizumabe } \\
\text { ANTICORPOS } \\
\text { MONOCLONAIS }\end{array}$ & $\begin{array}{c}32 \\
14 \\
3 \\
10 \\
1\end{array}$ & $\begin{array}{c}33,69 \\
14,74 \\
3,16 \\
10,53 \\
1,05\end{array}$ \\
\hline & $\begin{array}{l}\text { Cetuximabe } \\
\text { Denosumabe }\end{array}$ & $\begin{array}{l}1 \\
3\end{array}$ & $\begin{array}{l}1,05 \\
3,16\end{array}$ \\
\hline & $\begin{array}{c}\text { INIBIDORES } \\
\text { DA PROTEÍNA } \\
\text { QUINASE } \\
\text { Imatinibe } \\
\text { Pazopanibe }\end{array}$ & $\begin{array}{l}5 \\
3 \\
2\end{array}$ & $\begin{array}{l}5,27 \\
3,16 \\
2,11\end{array}$ \\
\hline
\end{tabular}

$\mathrm{Na}$ Tabela 2, apresenta-se a distribuição dos principais tratamentos com os imunoterápicos conforme a topografia do câncer e os tipos histológicos. Observa-se que 100\% dos pacientes tratados com BCG apresentavam câncer de bexiga; destes, 86 (27\%) eram carcinoma papilar de células transicionais. Nota-se, ainda, que três imunoterápicos foram utilizados em tipos diferentes de câncer. $\mathrm{O}$ interferon alfa 2-B, no câncer de rim e no melanoma; o denosumabe, no câncer de mama e no mieloma múltiplo; e o imatinibe, no tumor gastrointestinal e na leucemia mieloide crônica.

O quantitativo das imunoterapias em oncologia realizadas na unidade hospitalar analisada, a cada biênio da última década, está ilustrado na Figura 1. Pôde-se observar um aumento na utilização de imunoterápicos ao longo da década, bem como a incorporação de novos fármacos com o avanço dos anos, resultando em uma maior variedade de tratamentos realizados.

Na Tabela 3, apresentam-se a relação de óbitos durante o tratamento com imunoterápicos e o custeio deste entre os anos de 2010 a 2019. Observa-se que o imatinibe e o pazopanibe foram os medicamentos de maior porcentagem de óbitos. Nota-se, ainda, que, de um total de 51 pacientes em tratamento com BCG, apenas um paciente veio a óbito. Além disso, 77,9\% dos imunoterápicos utilizados foram custeados pelo Sistema Único de Saúde (SUS). Os medicamentos bevacizumabe, cetuximabe e denosumabe foram $100 \%$ financiados por não SUS.

O tempo de tratamento da maioria dos pacientes que utilizaram algum imunoterápico se manteve próximo de dez meses, sendo menor para os pacientes que se trataram com BCG (Figura 2). Além disso, o trastuzumabe foi o que obteve a maior variaçáo no tempo total de tratamento.

\section{DISCUSSÃO}

A imunoterapia é uma área recente e em crescimento, produto das muitas descobertas inovadoras em imunologia e terapia do câncer no último século. No entanto, apesar desse avanço, ainda existem obstáculos para o campo dessa terapêutica, entre eles, a incapacidade de prever a efetividade do tratamento; a necessidade de biomarcadores adicionais; o desenvolvimento de resistência; a falta de desenhos de estudos clínicos otimizados para determinar a eficácia; e os altos custos desse tratamento ${ }^{7}$.

Observou-se que, entre 2010 e 2019, 4,9\% dos pacientes com câncer na região receberam imunoterapêuticos. Estes ainda não são a primeira linha de tratamento para as neoplasias, mas, quando assim usados, não são feitos isoladamente, sendo associados a outros medicamentos. Para que isso ocorra, faz-se necessária a demonstração de eficácia comparável ou superior e toxicidade reduzida em 
Tabela 2. Distribuição dos principais tratamentos com imunoterápicos, conforme os tipos de câncer e tipos histológicos dos pacientes tratados na unidade hospitalar para tratamento oncológico, Barbacena-MG, 2010-2019 (n=78)

\begin{tabular}{|c|c|c|c|}
\hline Imunoterápico & $\begin{array}{l}\text { Tipo de câncer } \\
\text { n (\%) }\end{array}$ & Tipo histológico & n (\%) \\
\hline \multirow{3}{*}{ BCG } & \multirow{3}{*}{$\begin{array}{c}\text { Bexiga } \\
51(100,00)\end{array}$} & $\begin{array}{c}\text { Carcinoma papilar de células } \\
\text { transicionais }\end{array}$ & $44(86,27)$ \\
\hline & & Carcinoma de células transicionais & $6(11,76)$ \\
\hline & & Carcinoma papilar & $1(1,96)$ \\
\hline \multirow{3}{*}{ Interferon alfa 2-B } & \multirow{2}{*}{ $6(85,71)$} & Adenocarcinoma de células claras & $4(57,14)$ \\
\hline & & Carcinoma de células renais & $2(28,57)$ \\
\hline & $\begin{array}{l}\text { Melanoma } \\
1(14,29)\end{array}$ & Melanoma maligno & $1(14,29)$ \\
\hline \multirow{5}{*}{ Trastuzumabe } & \multirow{5}{*}{$\begin{array}{c}\text { Mama } \\
14(100,00)\end{array}$} & Carcinoma ductal infiltrante & $10(71,43)$ \\
\hline & & Adenocarcinoma ductal & $1(7,14)$ \\
\hline & & Carcinoma lobular & $1(7,14)$ \\
\hline & & Carcinoma ductular infiltrante & $1(7,14)$ \\
\hline & & Doença de Paget mamária & $1(7,14)$ \\
\hline \multirow{2}{*}{ Denosumabe } & $\begin{array}{c}\text { Mama } \\
2(66,67)\end{array}$ & Carcinoma ductal infiltrante & $2(66,67)$ \\
\hline & $\begin{array}{l}\text { Mieloma múltiplo } \\
1(33,33)\end{array}$ & Mieloma plasmocítico & $1(33,33)$ \\
\hline \multirow{2}{*}{ Imatinibe } & $\begin{array}{c}\text { Tumor estromal } \\
\text { gastrointestinal } \\
1(33,33)\end{array}$ & Sarcoma estromal gastrointestinal & $1(33,33)$ \\
\hline & $\begin{array}{l}\text { Leucemia mieloide crônica } \\
22(66,67)\end{array}$ & $\begin{array}{l}\text { Leucemia mieloide crônica, } \\
\text { granulocítica (SOE) }\end{array}$ & $2(66,67)$ \\
\hline
\end{tabular}

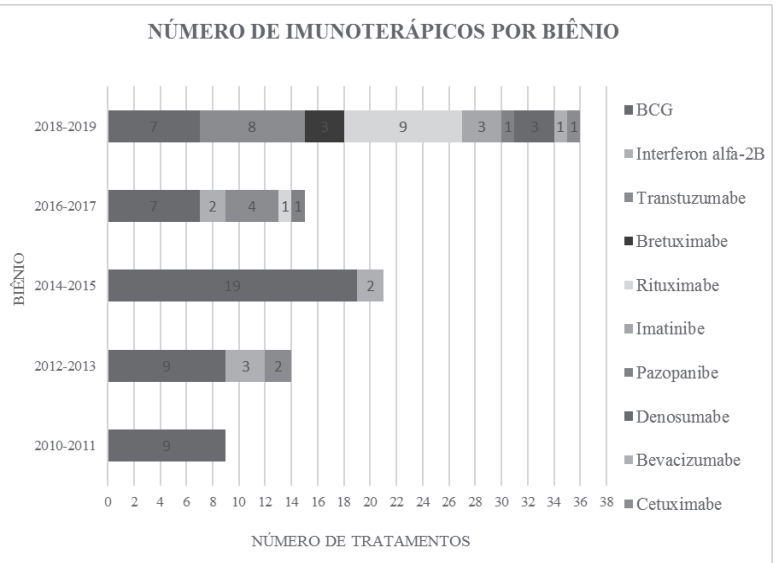

Figura 1. Quantitativo de tratamentos com imunoterápicos realizados, a cada biênio da década de 2010-2019, na unidade hospitalar para tratamento oncológico, Barbacena-MG ( $\mathrm{n}=95)$
Tabela 3. Óbitos ( $n=90)$ durante o tratamento com imunoterápicos e custeio do tratamento dos pacientes tratados na unidade hospitalar para tratamento oncológico, Barbacena-MG, 2010-2019

\begin{tabular}{cccc}
\hline Imunoterápico & $\begin{array}{c}\text { Óbitos } \mathbf{n} \\
(\%)\end{array}$ & SUS & Não SUS \\
\hline BCG & $1(1,96)$ & $43(84,31)$ & $8(15,69)$ \\
$\begin{array}{c}\text { Interferon } \\
\text { alfa-2B }\end{array}$ & $4(57,14)$ & $6(85,71)$ & $1(14,29)$ \\
$\begin{array}{c}\text { Trastuzumabe } \\
\text { Brentuximabe }\end{array}$ & $1(14,29)$ & $11(78,57)$ & $3(21,43)$ \\
Rituximabe & $3(30,0)$ & $8(80,00)$ & $2(20,0)$ \\
Bevacizumabe & 0 & 0 & $1(100,0)$ \\
Cetuximabe & 0 & 0 & $1(100,0)$ \\
Denosumabe & 0 & 0 & $3(100,0)$ \\
Imatinibe & $3(100,0)$ & $3(100,0)$ & 0 \\
Pazopanibe & $2(100,0)$ & $2(100,0)$ & 0 \\
\hline TOTAL & $\mathbf{1 6 ( 1 7 , 8 )}$ & $\mathbf{7 4}(\mathbf{7 7 , 9 )}$ & $\mathbf{2 1 ( 2 2 , 1 )}$ \\
\hline
\end{tabular}




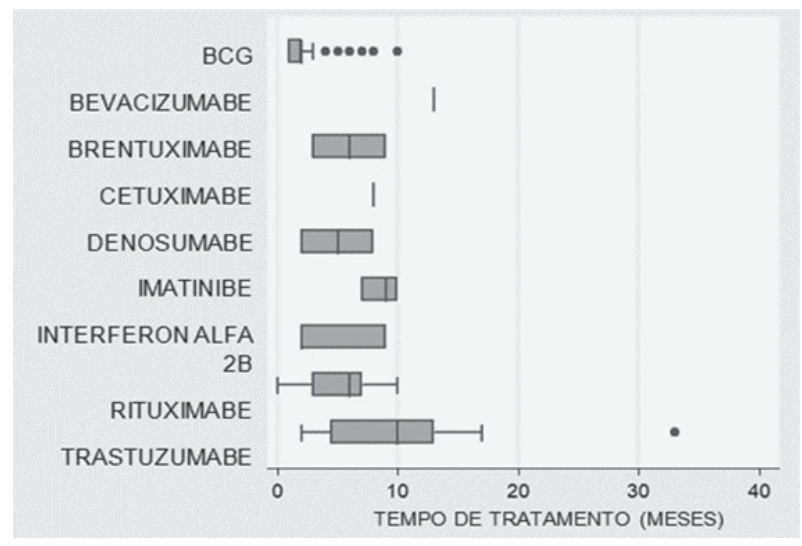

Figura 2. Tempo de tratamento em meses, relativo a cada imunoterápico utilizado por pacientes que concluíram o tratamento com imunoterápico no Setor de Oncologia do Hospital Ibiapaba, Barbacena-MG, 2010-2019 (n=95)

relação aos atuais agentes de primeira linha ${ }^{7}$. Além disso, existe uma lacuna entre a aprovação dos medicamentos em outros países e sua entrada no Brasil, o que promove um atraso na disponibilidade de imunoterapêuticos no país. Quando aprovados pela Agência Nacional de Vigilância Sanitária (Anvisa), os medicamentos oncológicos de administração intravenosa têm cobertura obrigatória pelos convênios privados. Para incorporação no SUS, devem ainda passar pela Avaliação de Tecnologias em Saúde (ATS) e serem aprovados pela Comissão Nacional para Incorporação de Tecnologias (Conitec), para então figurar no Registro Nacional de Medicamentos Essenciais (Rename). Por isso, há diferenças entre o que é registrado e o que é utilizado em sistemas de saúde privado, em relação ao que é ofertado pelo SUS 7 .

Os cânceres mais tratados com imunoterapêuticos em Barbacena-MG, durante o período estudado, foram o de bexiga, mama e linfoma, com maior frequência em pacientes acima de 50 anos. Esse dado corrobora as estatísticas do INCA, onde estes tumores são mais prevalentes a partir dessa faixa etária ${ }^{2}$. A idade média de diagnóstico para o câncer de bexiga é de 72 anos, sendo mais de $70 \%$ dos pacientes diagnosticados com o subtipo não invasivo, o que reforça os achados do presente estudo $^{11}$. Já para o câncer de mama HER-2 positivo, aquele responsivo à imunoterapia, as estimativas encontram-se em torno de 15\% para mulheres com idade superior a 50 anos $^{12}$. Em relaçáo ao linfoma, a média da idade ao diagnóstico para o não Hodgkin é de 67 anos, sendo $98 \%$ dos casos diagnosticados em adultos. Segundo Miller et al. ${ }^{11}$, aproximadamente $43 \%$ dos pacientes com esse tipo de câncer recebem quimioterapia associada à imunoterapia, a exemplo do rituximabe.

O quantitativo de imunoterápicos bem como a variedade de medicamentos utilizados aumentaram ao longo da década de 2010-2019 em Barbacena-MG. Até 2015, a imunoterapia na cidade se concentrava na utilizaçáo de BCG e interferon alfa-2B, com apenas dois pacientes utilizando trastuzumabe. A partir de 2016, um maior número de fármacos foi incorporado, a exemplo do rituximabe e do pazopanibe. E finalmente, nos anos de 2018-2019, foram incluídos o brentuximabe, o imatinibe, o denosumabe, o bevacizumabe e o cetuximabe, totalizando a realização de dez tipos diferentes de imunoterapias. $\mathrm{O}$ aumento da utilização de imunoterápicos ao longo da década em Barbacena-MG acompanha uma tendência mundial e reflete a necessidade de diversificação nos tratamentos oncológicos e a concentração no desenvolvimento de terapias alvo-específicas, as quais têm se mostrado mais efetivas e seguras em comparação às terapias convencionais, com diminuição do risco de recorrência da doença por meio da estimulação de uma memória antitumoral ${ }^{8,13}$. Provavelmente, ao longo do tempo, com o avanço e crescimento dos trabalhos e pesquisas nessa área, o uso da imunoterapia, tanto de forma isolada quanto em conjunto, poderá proporcionar um tratamento personalizado e melhorar a sobrevida global e livre de progressáo de muitos pacientes ${ }^{14}$. Uma vez consolidados como evidências, os resultados dessas pesquisas consistentes irão contribuir para a incorporação ainda mais de imunoterapêuticos pelo SUS, ampliando o acesso a essa classe de medicamentos.

Entre os imunoterápicos utilizados, destacam-se os modificadores biológicos, os anticorpos monoclonais e os inibidores da proteína quinase, tendo como principais representantes, respectivamente, o BCG, o trastuzumabe e o imatinibe. $\mathrm{O}$ primeiro agente aprovado mundialmente foi o modificador biológico interferon-alfa 2 (IFN-a2) em 1986. O BCG foi aprovado pela Food and Drug Administration (FDA) em 1990, destinando-se a tratar o carcinoma de bexiga não invasivo em estágio Tis, $\mathrm{Ta}$ e $\mathrm{T} 1{ }^{15}$, tendo, no Brasil, sua primeira utilização como tratamento de câncer em $2006^{16}$. Já os anticorpos monoclonais são proteínas modificadas derivadas de um mesmo clone de linfócito $\mathrm{B}$, destinadas a segmentar uma parte específica de sinais desregulados nas vias de transdução no câncer ou interferir com processos imunológicos ${ }^{3}$. Em 1999, foi aprovada a utilização do anticorpo monoclonal trastuzumabe pelo FDA para tratamento do câncer de mama metastático. Contudo, somente em 2006, tal medicamento foi internacionalmente indicado como tratamento adjuvante para esse tipo de câncer ${ }^{17}$. No Brasil, apenas em 2017, foi introduzido pelo SUS como tratamento de primeira linha para o câncer de mama HER-2 positivo metastático ${ }^{18}$. Por fim, a classe dos inibidores de proteína quinase, a exemplo do imatinibe, age prejudicando a proliferaçáo celular e a apoptose, uma vez que evita a transdução de sinais energéticos ${ }^{19}$. Em 2001, foi 
introduzido para uso em instituiçôes públicas brasileiras ${ }^{20}$, no mesmo ano em que foi aprovado pelo $\mathrm{FDA}^{21}$.

$\mathrm{Na}$ amostra analisada, houve $17,8 \%$ de mortes durante o tratamento. Em virtude dos poucos pacientes em uso desses fármacos, não foi possível avaliar a relação entre efetividade da estratégia terapêutica e mortalidade. Os óbitos provavelmente não estavam relacionados exclusivamente ao uso desses imunoterápicos em si, mas também ao tipo histológico do tumor, ao estadiamento, à idade e às comorbidades associadas.

De todos os tratamentos realizados no período, $77,9 \%$ foram custeados pelo SUS. Os efeitos dos custos de imunoterapêuticos no sistema público de saúde têm sido extensivamente discutidos e precisam ser considerados com atençáa ${ }^{7}$. As despesas das terapias contra o câncer estáo crescendo exponencialmente e projeta-se, para os próximos anos, um quadro que pode colocar o acesso ao tratamento oncológico sob ameaça. Com os fármacos de alto custo, o desafio de sustentabilidade do acesso a essas medicaçóes deixou de ser exclusivo de países em desenvolvimento, sendo uma questão complexa hoje, inclusive para os países desenvolvidos. No Brasil, os fármacos biológicos já são responsáveis por um percentual elevado das compras do Ministério da Saúde, em razão não apenas da adoção crescente desses produtos como opção terapêutica, mas também do alto valor agregado dessa classe de medicamentos ${ }^{8}$.

O BCG apresentou mediana de tratamento de três meses, condizente com a literatura atual ${ }^{22}$. Em uma metanálise realizada por Niraula e Gyawali²3, foi demonstrado que o trastuzumabe pode ser utilizado por nove semanas, seis meses ou um ano, a depender do protocolo adotado, corroborando a variaçáo encontrada no presente estudo ${ }^{23}$. Já a mediana no tempo de tratamento com rituximabe foi de sete meses, o que corresponde ao encontrado em outros países, onde a média da utilizaçáo desse imunoterápico ficou entre quatro semanas e 12 meses $^{24}$. As variaçóes observadas no tempo de tratamento reforçam a individualização da imunoterapia, como resultado da combinação de resposta terapêutica e toxicidade em cada paciente.

Apenas pacientes diagnosticados e tratados na unidade hospitalar de referência oncológica da região de saúde foram incluídos no estudo, o que foi um fator limitante para uma análise que representasse com maior veracidade o uso de tais medicamentos na Regiáo em questão, já que presume-se que indivíduos de outros locais referenciados para a unidade poderiam também estar em uso dessa terapia. Por outro lado, este estudo avaliou os imunoterápicos incorporados na rotina da instituição em Barbacena-MG, abordagem inédita e ainda insipiente na literatura brasileira até o presente.

\section{CONCLUSÃO}

$\mathrm{Na}$ última década, 4,9\% dos pacientes oncológicos realizaram tratamento com imunoterapêuticos em Barbacena-MG. A maioria dos pacientes era maior de 50 anos; o tratamento mais prevalente foi para o câncer de bexiga com o BCG; e 17,8\% dos pacientes foram a óbito durante o tratamento. Ao longo dos anos, novos imunoterapêuticos foram incorporados, com destaque para os dois últimos anos: 2018 e 2019, nos quais foram incorporados cinco dos dez imunoterápicos utilizados em toda a década. $\mathrm{O}$ aperfeiçoamento e a pesquisa de novas opçôes terapêuticas em oncologia se fazem contínuos e justificáveis, a fim de que tal tratamento seja disponibilizado em amplo espectro para a promoção da saúde com qualidade.

\section{CONTRIBUIÇÕES}

Todos os autores contribuíram igualmente na concepção e/ou no planejamento do estudo; na obtençáo, análise e interpretação dos dados; assim como na redação e revisão crítica; e aprovaram a versão final a ser publicada.

\section{DECLARAÇÃO DE CONFLITO DE INTERESSES}

Nada a declarar.

\section{FONTES DE FINANCIAMENTO}

Não há.

\section{REFERÊNCIAS}

1. World Health Organization [Internet]. Geneva: WHO; c2020. News release, Early cancer diagnosis saves lives, cuts treatment costs; 2017 Feb 3 [cited 2020 Apr 6]. Available from: https://www.who.int/en/news-room/ detail/03-02-2017-early-cancer-diagnosis-saves-livescuts-treatment-costs

2. Instituto Nacional de Câncer José Alencar Gomes da Silva. Estimativa 2020: incidência de câncer no Brasil [Internet]. Rio de Janeiro: INCA; 2019 [acesso 2020 abr 25]. Disponível em: https://www.inca.gov.br/sites/ ufu.sti.inca.local/files/media/document/estimativa-2020incidencia-de-cancer-no-brasil.pdf

3. Zhang $\mathrm{H}$, Chen J. Current status and future directions of cancer immunotherapy. J Cancer. 2018;9(10):1773-81. doi: https://doi.org/10.7150/jca.24577

4. Kaliks RA. An update on clinical oncology for the non-oncologist. Einstein (São Paulo). 2016;14(2):294-99. doi: https://doi.org/10.1590/ S1679-45082016MD3550 
5. Martinez VG, Park D, Acton SE. Immunotherapy: breaching the barriers for cancer treatment. Philos Trans R Soc Lond B Biol Sci. 2019;374(1779):20180214. doi: https://doi.org/10.1098/rstb.2018.0214

6. Teixeira HC, Dias LS, Menão TL, et al. Proteínas de checkpoint imunológico como novo alvo da imunoterapia contra o câncer: revisão da literatura. HU Rev. 2019;45(3):325-33. doi: https://doi.org/10.34019/19828047.2019.v45.28820

7. Ventola CL. Cancer immunotherapy, part 2: efficacy, safety, and other clinical considerations. P T [Internet]. 2017 [cited 2020 abr 25];42(7):452-63. Available from: https:/www.ncbi.nlm.nih.gov/pmc/articles/ PMC5481296/pdf/ptj4207452.pdf

8. Vidal TJ, Figueiredo TA, Pepe VLE. O mercado brasileiro de anticorpos monoclonais utilizados para o tratamento de câncer. Cad Saúde Pública. 2018;34(12):e00010918. doi: https://doi.org/10.1590/0102-311X00010918

9. Cidades@: sistema agregador de informações sobre os municípios e estados do Brasil [Internet]. Version 4.4.13. Rio de Janeiro: IBGE. c2017. Barbacena, MG; [acesso 2020 abr 22]. Disponível em: https://cidades.ibge.gov. $\mathrm{br} / \mathrm{brasil} / \mathrm{mg} /$ barbacena/panorama

10. Conselho Nacional de Saúde (BR). Resolução no 466, de 12 de dezembro de 2012. Diário Oficial da União, Brasília, DF; 2013 jun. 13. Seção I, p. 59.

11. Miller KD, Nogueira L, Mariotto AB, et al. Cancer treatment and survivorship statistics, 2019. CA Cancer J Clin. 2019;69(5):363-85. doi: https://doi.org/10.3322/ caac. 21565

12. Cronin KA, Harlan LC, Dodd KW, et al. Populationbased estimate of the prevalence of HER-2 positive breast cancer tumors for early stage patients in the US. Cancer Invest. 2010;28(9):963-8. doi: https://doi.org/10.3109 /07357907.2010.496759

13. Wayteck L, Breckpot K, Demeester J, et al. A personalized view on cancer immunotherapy. Cancer Lett. 2014;352(1):113-25. doi: https://doi.org/10.1016/j. canlet.2013.09.016

14. Stanculeanu DL, Daniela Z, Lazescu A, et al. Development of new immunotherapy treatments in different cancer types. J Med Life [Internet]. 2016 [cited 2020 maio 5];9(3):240-48. Available from: https://www. ncbi.nlm.nih.gov/pmc/articles/PMC5154307/pdf/ JMedLife-09-240.pdf Free full text article. PMCID: PMC 5154307.

15. Eno J. Immunotherapy through the years. J Adv Pract Oncol [Internet]. 2017;8(7):747-53. Available from: https://www.ncbi.nlm.nih.gov/pmc/articles/ PMC6188092/pdf/jadp-08-747.pdf Free full text article. PMCID: PMC 6188092.

16. Louvison MCP, Bersusa AAS, Bonfim JRA, et al. Imunoterapia com onco BCG para tratamento adjuvante de câncer superficial de bexiga: parecer técnico-científico
[Internet]. São Paulo: Instituto de Saúde; 2013 [acesso 2020 abr 6]. 31 p. Disponível em: http://www.saude. sp.gov.br/resources/instituto-de-saude/homepage/ nucleos/nucleo-de-analise-e-projetos-de-avaliacao-detecnologias-de-saude/ptc_onco_bcg_com_capa.pdf

17. Produtos Roche Químicos e Farmacêuticos S.A. Consulta Pública da CONITEC/SCTIE no 13/2017: recomendação sobre proposta de incorporação no SUS do medicamento pertuzumabe para o tratamento do câncer de mama HER2-positivo metastático em primeira linha de tratamento associado ao trastuzumabe e docetaxel [Internet]. São Paulo: Roche; 2017 abr 28 [acesso 2020 abr 6]. 10 p. Disponível em: http://formsus.datasus.gov. br/novoimgarq/31137/5672278_312373.pdf

18. Ministério da Saúde (BR). Trastuzumabe para o tratamento do câncer de mama HER2-positivo metastático em primeira linha de tratamento [Internet]. Brasília, DF: CONITEC; 2017 abr [acesso 2020 abr 6]. 71 p. Disponível em: http://conitec.gov.br/ images/Relatorios/2017/Relatorio_Trastuzumabe_CA_ MamaMetastatico_CP.pdf

19. Lopes NR, Abreu, MTCL. Inibidores de tirosino quinase na leucemia mieloide crônica. Rev Bras Hematol Hemoter. 2009;31(6):449-53. http://doi.org/10.1590/ S1516-84842009005000089

20. Silveira CAP. Resposta ao tratamento com mesilato de Imatinibe nos portadores de Leucemia Mielóide Crônica do Hospital de Base do Distrito Federal [tese na Internet]. Brasília, DF: Universidade de Brasília; 2011 [acesso 2020 maio 5]. 111 p. Disponível em: https:// repositorio.unb.br/bitstream/10482/7596/1/2011_ CarlosAlbertoPintoSilveira.pdf

21. Azevedo LD, Bastos MM, Oliveira AP, et al. Sínteses e propriedades de fármacos inibidores da tirosina quinase bcr-abl, utilizados no tratamento da leucemia mieloide crônica. Quím Nova. 2017;40(7):791-809. doi: https:// doi.org/10.21577/0100-4042.20170027

22. Fuge O, Vasdev N, Allchorne P, et al. Immunotherapy for bladder cancer. Res Rep Urol. 2015;7:65-79. doi: https://doi.org/10.2147/RRU.S63447

23. Niraula S, Gyawali B. Optimal duration of adjuvant trastuzumab in treatment of early breast cancer: a meta-analysis of randomized controlled trials. Breast Cancer Res Treat. 2019;173(1):103-9. doi: https://doi. org/10.1007/s10549-018-4967-8

24. Coiffier B. Rituximab therapy in malignant lymphoma. Oncogene. 2007;26:3603-13. doi: https://doi. org/10.1038/sj.onc. 1210376 